\title{
SMALL CONGRUENCES AND CONCRETENESS
}

\author{
MAGDALENA VELEBILOVÁ
}

(Communicated by Maurice Auslander)

\begin{abstract}
Let $\underline{K}$ be a concrete category and $\sim$ a congruence on $\underline{K}$. Let $\sim$ be generated by a class $\mathscr{M}=\mathscr{M}_{1} \cup \mathscr{M}_{2}$ of pairs of $\underline{K}$-morphisms such that $\left\{\operatorname{dom} f ;(\exists g)\left((f, g) \in \mathscr{M}_{1}\right)\right\}$ and $\left\{\operatorname{rng} f ;(\exists g)\left((f, g) \in \mathscr{M}_{2}\right)\right\}$ are small sets. Then $\underline{K} / \sim$ is concrete. Consequently, if $\sim$ is generated by a small set of pairs of morphisms, then $\underline{K} / \sim$ is concrete.
\end{abstract}

By the Kučera theorem [5], every category is a factorization of a concrete category. V. Koubek and J. Reiterman in [4] showed that factorizing a full subcategory of Set we obtain a concrete category again. In fact, the reason is that there are only very "simple" congruences on the full subcategory of Set and they cannot spoil the concreteness of the factor.

This leads to a natural question: How rich must the congruence spoiling concreteness be? For example, can it be generated by one pair of morphisms? The answer is no; moreover, there is a wide class of congruences that preserves concreteness, because they are small in some sense. In particular, any congruence generated by a small set of pairs of morphisms preserves concreteness. For every congruence on a full subcategory of Set that has the mentioned property [4], any factor of such category is concrete.

I am indebted to professor Verra Trnková for turning my attention to this problem and for many valuable comments.

Conventions. By a category we always mean a locally small one. If $\underline{K}$ is a category, then $\underline{K}^{m}$ denotes the class of all $\underline{K}$-morphisms.

We work in the Gödel-Bernays set theory with the axiom of choice. We denote by On the class of all ordinal numbers and by $\mathrm{Cn}$ the class of all cardinal numbers.

\section{CONGRUences}

By a congruence $\sim$ on $\underline{K}$ we mean an equivalence on $\underline{K}^{m}$ satisfying

(i) if $f \sim g$, then $\operatorname{dom} f=\operatorname{dom} g$ and $\operatorname{rng} f=\operatorname{rng} g$,

(ii) if $f \sim g$, then $f \circ h \sim g \circ h$ and $k \circ f \sim k \circ g$, whenever the composition makes sense.

Received by the editors July 17, 1990 and, in revised form, November 27, 1990.

1980 Mathematics Subject Classification (1985 Revision). Primary 18B05, 18A32.

Key words and phrases. Concrete category, congruence. 
If $\sim$ is a congruence on $\underline{K}$, then the category $\underline{K} / \sim$ has the same objects as $\underline{K}$ and the $\underline{K} / \sim$-morphisms are the classes $[f]_{\sim}$ for all $f \in \underline{K}$.

Let $\mathscr{M}$ be a class of pairs of $K$-morphisms such that if $(f, g) \in \mathscr{M}$, then $\operatorname{dom} f=\operatorname{dom} g$ and $\operatorname{rng} f=\operatorname{rng} g$. The smallest congruence on $\underline{K}$ containing $\mathscr{M}$ is called the congruence generated by $\mathscr{M}$. Let $\mathscr{M}=\left\{\left(f_{t}, g_{t}\right) ; t \in T\right\}$ have the property $\left(f_{t}, g_{t}\right) \in \mathscr{M} \Rightarrow\left(g_{t}, f_{t}\right) \in \mathscr{M}$. Put $f \dot{\sim} g$ iff there are $t \in T$, $k, h \in \underline{K}^{m}$ such that $f=h \circ f_{t} \circ k$ and $g=h \circ g_{t} \circ k$. It is easy to verify that $\sim$ is the reflexive and transitive closure of $\dot{\sim}$.

1.1. Definitions. A congruence $\sim$ is called $d$-small $(r$-small, resp.) if there is a class $\mathscr{M}$ such that $\mathscr{M}=\left\{\left(f_{t}, g_{t}\right) ; t \in T\right\}$ generating $\sim$ in such a way that $\left\{\operatorname{dom} f_{t} ; t \in T\right\}\left(\left\{\operatorname{rng} f_{t} ; t \in T\right\}\right.$, resp. $)$ is a small set. A small congruence is a congruence generated by a small set of pairs of morphisms.

Let $\left\{\sim_{i} ; i \in I\right\}$ be a set of congruences on $\underline{K}$. By its join, $\bigvee_{I} \sim_{i}$, we mean the congruence generated by $\bigcup_{I} \sim_{i}$.

1.2. Lemma. $\bigvee_{I} \sim_{i}$ is the transitive closure of $\bigcup_{I} \sim_{i}$.

Proof. For $f, g \in \underline{K}^{m}$, put $f \sim g$ iff there are $m_{1}, \ldots, m_{n} \in \underline{K}^{m}$ such that $f=m_{1} \sim_{i_{1}} \cdots \sim_{i_{n-1}} m_{n}=g$ and $i_{j} \in I$ for every $j \in\{1, \ldots, n-1\}$. Clearly $\sim$ is an equivalence. If $\operatorname{rng} h=\operatorname{dom} f$, then $f \circ h=m_{1} \circ h \sim_{i_{1}}$ $\cdots \sim_{i_{n-1}} m_{n} \circ h=g \circ h$, hence $f \circ h \sim g \circ h$. If $\operatorname{dom} h=\operatorname{rng} f$, then $h \circ f=h \circ m_{1} \sim_{i_{1}} \cdots \sim_{i_{n-1}} h \circ m_{n}=h \circ g$, hence $h \circ f \sim h \circ g$. Thus $\sim$ is a congruence and it is $\bigvee_{I} \sim_{i}$.

Suppose $\sim_{1}$ and $\sim_{2}$ are congruences on $\underline{K}$ and $\sim$ is their join. Consider the category $\underline{K}_{1}=\underline{K} / \sim_{1}$ and put $[f]_{\sim_{1}} \dot{\sim}_{2}[g]_{\sim_{1}}$ iff for some $f^{\prime} \in[f]_{\sim_{1}}$, $g^{\prime} \in[g]_{\sim_{1}} f^{\prime} \sim_{2} g^{\prime}$ holds. Let $\approx_{2}$ be the transitive closure of $\dot{\sim}_{2}$.

\subsection{Proposition. Relation defined above is a congruence and $\underline{K}_{1} / \approx_{2} \cong \underline{K} / \sim$.}

Proof. Obivously $\approx_{2}$ is an equivalence. Let $\operatorname{rng} h=\operatorname{dom} f$. If $[f]_{\sim_{1}} \dot{\sim}_{2}[g]_{\sim_{1}}$, then there are $f^{\prime}, g^{\prime}$ such that $f \sim_{1} f^{\prime}, f^{\prime} \sim_{2} g^{\prime}, g^{\prime} \sim_{1} g$, hence $f \circ h \sim_{1}$ $f^{\prime} \circ h, f^{\prime} \circ h \sim_{2} g^{\prime} \circ h, g^{\prime} \circ h \sim_{1} g \circ h$, thus $[f]_{\sim_{1}} \circ[h]_{\sim_{1}}=[f \circ h]_{\sim_{1}} \dot{\sim}_{2}[g \circ h]_{\sim_{1}}=$ $[g]_{\sim_{1}} \circ[h]_{\sim_{1}}$. If $[f]_{\sim_{1}} \approx_{2}[g]_{\sim_{1}}$, then $[f]_{\sim_{1}}=\left[m_{1}\right]_{\sim_{1}} \dot{\sim}_{2} \cdots \dot{\sim}_{2}\left[m_{n}\right]_{\sim_{1}}=[g]_{\sim_{1}}$, hence $[f]_{\sim_{1}} \circ[h]_{\sim_{1}} \approx_{2}[g]_{\approx_{1}} \circ[h]_{\sim_{1}}$. Similarly for $\operatorname{dom} h=\operatorname{rng} f$. There is a functor $F: \underline{K} / \sim \rightarrow \underline{K}_{1} / \approx_{2}$ defined $F X=X$ on objects and $F[f]_{\sim}=$ $\left[[f]_{\sim_{1}}\right]_{\approx_{2}}$ on morphisms. By $1.2, F$ is defined correctly. It is easy to verify that $F$ is faithful, and as it is clearly full and one-to-one on objects it is an isomorphism.

Clearly if $\sim_{2}$ is $d$-small or $r$-small, then so is $\approx_{2}$.

\section{Combinatorial tool}

Recall that if $\kappa, \lambda, \mu, r$ are cardinal numbers, then the abbreviation $\kappa \rightarrow(\lambda)_{\mu}^{r}$ means: For any set $A$ with $\operatorname{card} A=\kappa$ and for any mapping $\omega:[A]^{r} \rightarrow \mu$ there is a subset $A^{\prime}$ of $A$ and $i \in \mu$ such that card $A^{\prime}=\lambda$ and $\omega\left([A]^{r}\right)=\{i\}$.

2.1. Lemma. Let $\mathscr{A}$ be a proper class and I a small set. Then for any mapping $\omega:[\mathscr{A}]^{2} \rightarrow I$, there is some $i \in I$ having the following property: For every $\lambda \in C n$ there is a set $A_{\lambda} \subseteq \mathscr{A}$ such that card $A_{\lambda}=\lambda$ and $\omega\left(\left[A_{\lambda}\right]^{2}\right)=\{i\}$. 
Proof. By the Theorem 4 in [1], for every infinite cardinal number $\kappa,\left(2^{\kappa}\right)^{+} \rightarrow$ $\left(\kappa^{+}\right)_{\kappa}^{2}$ holds. It follows immediately that for every $\lambda, \mu \in C n$ there exists $\kappa \in C n$ such that $\kappa \rightarrow(\lambda)_{\mu}^{2}$. The rest of the proof is obvious.

\section{ISBELL $n$-CONDITION}

By the Isbell condition we mean the condition for category to be concrete, stated by J. R. Isbell [3] as necessary and proved by P. J. Freyd [2] as sufficient. We use its modified form.

3.1. Definitions. Let $A, X_{1}, \ldots, X_{n}$ be $\underline{K}$-objects. The diagram of the type

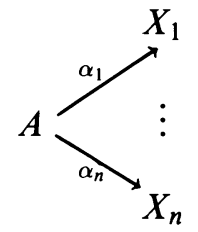

is called the $n$-span over $X_{1}, \ldots, X_{n}$ and is denoted by $\left(A, \alpha_{i}\right)$. Dually we can define the $n$-cospan $\left(\gamma_{i}, Y\right)$ as the diagram of the type

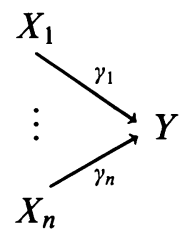

We say that $n$-spans (over $\left.X_{1}, \ldots, X_{n}\right)\left(A, \alpha_{i}\right)$ and $\left(B, \beta_{i}\right)$ are equivalent iff for every $n$-cospan $\left(\gamma_{i}, Y\right)$ holds: $\forall i, j \in\{1, \ldots, n\}, i \neq j: \gamma_{i} \circ \alpha_{i}=\gamma_{i} \circ \alpha_{i}$ iff $\gamma_{i} \circ \boldsymbol{\beta}_{i}=\gamma_{i} \circ \boldsymbol{\beta}_{j}$. Let us denote this equivalence by $\equiv$.

We say that $\underline{K}$ satisfies the Isbell $n$-condition if for every $n$-tuple $X_{1}, \ldots, X_{n}$ of $\underline{K}$-objects there is a choice set (denoted by $M_{x_{1}, \ldots, x_{n}}$ ) of $n$-spans over $X_{1}, \ldots, X_{n}$ such that every $n$-span over $X_{1}, \ldots, X_{n}$ is equivalent to some $n$-span in $M_{x_{1}, \ldots, x_{n}}$.

Clearly the Isbell 2-condition is the well-known Isbell condition.

3.2. Proposition. A category $\underline{K}$ is concrete iff it satisfies the Isbell $n$-condition for every $n \geq 2$.

Proof. $\Leftarrow$ is obvious as $\underline{K}$ then satisfies the Isbell condition [3].

$\Rightarrow$ Let there exist $n \geq 2$ such that $\underline{K}$ does not satisfy the Isbell $n$-condition. Thus there exists an $n$-tuple $X_{1}, \ldots, X_{n}$ of $\underline{K}$-objects and a proper class $\mathscr{A}$ of pairwise nonequivalent $n$-spans over $X_{1}, \ldots, X_{n}$. That means, for every $\left(A, \alpha_{i}\right),\left(B, \beta_{i}\right)$ in $\mathscr{A}$, there are $i, j \in\{1, \ldots, n\}$ and an $n$-cospan $\left(\gamma_{i}, Y\right)$ such that

$$
\gamma_{i} \circ \alpha_{i}=\gamma_{i} \circ \alpha_{j} \quad \text { iff } \gamma_{i} \circ \beta_{i} \neq \gamma_{j} \circ \beta_{j} .
$$

There is a mapping

$$
\begin{gathered}
\omega:[\mathscr{A}]^{2} \rightarrow[\{1, \ldots, n\}]^{2}, \\
\left\{\left(A, \alpha_{i}\right),\left(B, \beta_{i}\right)\right\} \mapsto \text { some }\{i, j\} \text { satisfying }(*) .
\end{gathered}
$$


By 2.1, there is a pair $\{i, j\}$ such that for every $\lambda \in C n$ there is $A_{\lambda} \subseteq \mathscr{A}$, card $A_{\lambda}=\lambda$, and $\omega\left(\left[A_{\lambda}\right]^{2}\right)=\{i, j\}$. Thus $\left\{\left(A, \alpha_{i}, \alpha_{j}\right) ;\left(A, \alpha_{i}\right) \in A_{\lambda}\right\}$ is a set of nonequivalent 2-spans over $X_{i}, X_{j}$ with cardinality $\lambda$. So there cannot exist a choice set $M_{x_{i}, x_{j}}$ and $\underline{K}$ then does not satisfy the Isbell condition, hence $\underline{K}$ is not concrete.

\section{THE MAIN THEOREM}

4.1. Theorem. Let $\underline{K}$ be a concrete category, and let $\sim$ be a $d$-small congruence on $\underline{K}$. Then $\underline{K} / \sim$ is concrete.

Proof. Let $\underline{K} / \sim$ be nonconcrete. There must exist $\underline{K}$-objects $X, Y, Z_{i} \quad(i \in$ On) and for every $i, j \in \mathrm{On}(i \neq j)$ an object $Q_{i j}$ and morphisms $\left[\gamma_{i j}\right]_{\sim}: X \rightarrow$ $Q_{i j},\left[\delta_{i j}\right]_{\sim}: Y \rightarrow Q_{i j}$ such that $Q_{i j}=Q_{j i}, \gamma_{i j}=\gamma_{j i}, \delta_{i j}=\delta_{j i}$, and $\left[\gamma_{i j}\right]_{\sim} \circ$ $\left[\alpha_{i}\right]_{\sim}=\left[\delta_{i j}\right]_{\sim} \circ\left[\beta_{i}\right]_{\sim}$ iff $\left[\gamma_{i j}\right]_{\sim} \circ\left[\alpha_{j}\right]_{\sim} \neq\left[\delta_{i j}\right]_{\sim} \circ\left[\beta_{j}\right]_{\sim}:$

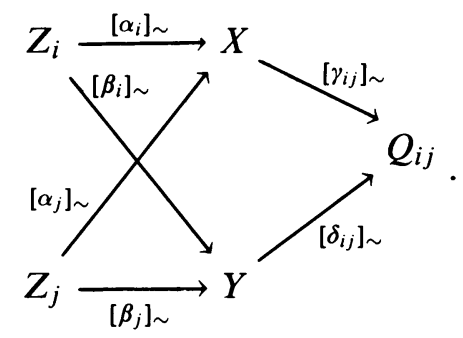

Now consider the diagram in $\underline{K}$ on the same objects as above but with morphisms $f$ instead of $[f]_{\sim}$. We obtain a proper class of 2-spans but as $\underline{K}$ is concrete, there must be only a set of nonequivalent 2-spans. Without loss of generality we can suppose that the whole class consists of equivalent 2-spans.

Obviously for every $i, j \in$ On with $i \neq j, \gamma_{i j} \circ \alpha_{i} \neq \delta_{i j} \circ \beta_{i}, \gamma_{i j} \circ \alpha_{j} \neq$ $\delta_{i j} \circ \beta_{j}$, and $\gamma_{i j} \circ \alpha_{i} \sim \delta_{i j} \circ \beta_{i}$ iff $\gamma_{i j} \circ \alpha_{j} \nsim \delta_{i j} \circ \beta_{j}$. Denote $\mathscr{P}=$ $\left\{(i, j) ; i, j \in\right.$ On,$\left.\gamma_{i j} \circ \alpha_{i} \sim \delta_{i j} \circ \beta_{i}\right\}$. Clearly $(i, j) \in \mathscr{P}$ iff $(j, i) \notin \mathscr{P}$. As $\sim$ is $d$-small there exists a class $\mathscr{M}$ of pairs of $K$-morphisms generating $\sim$ such that $\{\operatorname{dom} f ;(f, g) \in \mathscr{M}\}$ is a small set and if $(f, g) \in \mathscr{M}$ then $(g, f) \in \mathscr{M}$. So for every $(i, j) \in \mathscr{P}$ there exist morphisms $m_{i j}(1), \ldots, m_{i j}\left(n_{i j}\right), n_{i j} \geq 2$ such that $\gamma_{i j} \circ \alpha_{i}=m_{i j}(1) \dot{\sim} \cdots \dot{\sim} m_{i j}\left(n_{i j}\right)=\delta_{i j} \circ \beta_{i}$, or in other words, following $\S 1$,

$$
\begin{aligned}
\gamma_{i j} \circ \alpha_{i}= & h_{i j}(1) \circ f_{i j}(1) \circ k_{i j}(1) \dot{\sim} h_{i j}(1) \circ g_{i j}(1) \circ k_{i j}(1) \\
= & h_{i j}(2) \circ f_{i j}(2) \circ k_{i j}(2) \dot{\sim} \ldots \\
& \dot{\sim} h_{i j}\left(n_{i j}\right) \circ g_{i j}\left(n_{i j}\right) \circ k_{i j}\left(n_{i j}\right)=\delta_{i j} \circ \beta_{i},
\end{aligned}
$$

where $\left(f_{i j}(n), g_{i j}(n)\right) \in \mathscr{M}, k_{i j}(n): Z_{i} \rightarrow D_{i j}(n), f_{i j}(n), g_{i j}(n): D_{i j}(n) \rightarrow$ $R_{i j}(n), h_{i j}(n): R_{i j}(n) \rightarrow Q_{i j}$ for every $(i, j) \in \mathscr{P}, n \in\left\{1, \ldots, n_{i j}\right\}$.

Consider the set $\mathscr{D}=\left\{D_{i j}(n) ;(i, j) \in \mathscr{P}, n \in\left\{1, \ldots, n_{i j}\right\}\right\} \cup\{X, Y\}$. For $\underline{K}$ concrete, the Isbell $n$-condition is satisfied for each $n \geq 2$. Therefore for every $n$-tuple $\mathbb{X}=\left(X_{1}, \ldots, X_{n}\right) \quad\left(X_{1}, \ldots, X_{n} \in \mathscr{D}\right)$ there is a choice set $M_{X_{1}, \ldots, X_{n}}$ of $n$-spans over $X_{1}, \ldots, X_{n}$ (in the sense of $\S 3$ ); let $\lambda_{\mathrm{x}}=$ $\operatorname{card} M_{X_{1}, \ldots, X_{n}}$. Set $\lambda:=\sup \left\{\lambda_{\mathrm{x}} ; \mathbb{X}=\left(X_{1}, \ldots, X_{n}\right), X_{1}, \ldots, X_{n} \in \mathscr{D}\right\}, \kappa:=$ $\left(2^{\lambda}\right)^{+}$. Let $I$ be the set of all finite sequences of the type $\left(D_{i j}(1), \ldots, D_{i j}\left(n_{i j}\right)\right)$. 
There is a mapping

$$
\begin{aligned}
\omega:[\mathrm{On}]^{2} & \rightarrow I \\
\{i, j\} & \mapsto\left(D_{i j}(1), \ldots, D_{i j}\left(n_{i j}\right)\right) \quad \text { if }(i, j) \in \mathscr{P} .
\end{aligned}
$$

By 2.1., there is a subset $A \subseteq$ On,$\hat{n} \in \mathbb{N}$, and an $\hat{n}$-tuple $\left(D_{1}, \ldots, D_{\hat{n}}\right)$ such that $\operatorname{card} A=\kappa$ and for every $(i, j) \in P_{A}=\mathscr{P} \cap(A \times A)$,

$$
\left(D_{i j}(1), \ldots, D_{i j}\left(n_{i j}\right)\right)=\left(D_{1}, \ldots, D_{\hat{n}}\right) \text { holds } .
$$

Take the $(2 \hat{n}+2)$-tuple $\left(X, D_{1}, D_{1}, \ldots, D_{\hat{n}}, D_{\hat{n}}, Y\right)$. By assumptions there is at most $\lambda$ nonequivalent $(2 \hat{n}+2)$-spans over $\left(X, D_{1}, D_{1}, \ldots, D_{\hat{n}}, D_{\hat{n}}, Y\right)$. We show that it leads to a contradiction.

Consider all spans of the type $\left(\alpha_{i}, k_{i j}(1), k_{i j}(1), \ldots, k_{i j}(\hat{n}), k_{i j}(\hat{n}), \beta_{i}\right)$ (denoted by $\overline{(i, j)})$ for every $(i, j) \in P_{A}$.

First, we prove that if $(i, j),(j, l) \in P_{A}$, then $\overline{(i, j)} \not \equiv \overline{(j, l)}$. Suppose $\overline{(i, j)} \equiv \overline{(j, l)}$ and choose the cospan

$$
\left(\gamma_{i j}, h_{i j}(1) \circ f_{i j}(1), h_{i j}(1) \circ g_{i j}(1), \ldots, h_{i j}(\hat{n}) \circ f_{i j}(\hat{n}), h_{i j}(\hat{n}) \circ g_{i j}(\hat{n}), \delta_{i j}\right) \text {. }
$$

Then

$$
\begin{aligned}
\gamma_{i j} \circ \alpha_{j} & =h_{i j}(1) \circ f_{i j}(1) \circ k_{j l}(1) \dot{\sim} h_{i j}(1) \circ g_{i j}(1) \circ k_{j l}(1)=\cdots \\
& =h_{i j}(\hat{n}) \circ f_{i j}(\hat{n}) \circ k_{j l}(\hat{n}) \dot{\sim} h_{i j}(\hat{n}) \circ g_{i j}(\hat{n}) \circ k_{j l}(\hat{n})=\delta_{i j} \circ \beta_{j},
\end{aligned}
$$

and hence $\gamma_{i j} \circ \alpha_{j} \sim \delta_{i j} \circ \beta_{j}$-a contradiction. Now we prove that there cannot exist a choice set $M=M_{X, D_{1}, D_{1}, \ldots, D_{\hat{n}}, D_{\hat{n}}, Y}$ with card $M=\lambda$. Suppose the contrary.

Let $\pi: P_{A} \rightarrow M$ be a natural projection, i.e., $\pi(i, j)$ is a $(2 \hat{n}+2)$-span in $M$ equivalent with $\overline{(i, j)}$. Define $P_{i}=P_{A} \cap(\{i\} \times A)$ for each $i \in A$. Clearly $P_{A}=\bigcup_{i \in A} P_{i}$ is a disjoint union. As card $A=\kappa=\left(2^{\lambda}\right)^{+}$, there must be $i, j, \in A$ such that $\pi\left(P_{i}\right)=\pi\left(P_{j}\right)$. Without loss of generality let $(i, j) \in P_{i}$. Then there exists $l \in A$ such that $\pi(i, j)=\pi(j, l)$. Thus $\overline{(i, j)} \equiv \overline{(j, l)}$, a contradiction.

Hence $\underline{K} / \sim$ is a concrete category.

4.2. Corollary. For $\underline{K}$ a concrete category, $\sim$ a congruence on $\underline{K}$ the following holds:

(i) If $\sim$ is small, then $\underline{K} / \sim$ is concrete;

(ii) if $\sim$ is $r$-small, then $\underline{K} / \sim$ is concrete;

(iii) if $\sim$ is a join of $d$-small and $r$-small congruences, then $\underline{K} / \sim$ is concrete.

Proof. (i) and (ii) are obvious.

(iii) Let $\sim=\sim_{1} \vee \sim_{2}$ where $\sim_{1}$ is $d$-small and $\sim_{2}$ is $r$-small. $\underline{K} / \sim_{1}=\underline{K}_{1}$ is concrete by the Theorem and $\approx_{2}$ defined as in $\S 1$ is also $r$-small. For $\underline{K}_{1} / \approx_{2}$ concrete and $\underline{K} / \sim \cong \underline{K}_{1} / \approx_{2}, \underline{K} / \sim$ is concrete.

\section{REFERENCES}

1. P. Erdős and R. Rado, A partition calculus in set theory, Bull. Amer. Math. Soc. 62 (1956), 427-489.

2. P. J. Freyd, Concreteness, J. Pure Appl. Algebra 3 (1973), 171-191.

3. J. R. Isbell, Two set-theoretical theorems in categories, Fund. Math. 53 (1963), 43-49. 
4. V. Koubek and J. Reiterman, Factor categories of the category of sets: Description and concreteness, J. Pure Appl. Algebra 4 (1974), 71-77.

5. L. Kučera, Every category is a factorization of a concrete one, J. Pure Appl. Algebra 1 (1971), 373-376.

Charles University, Sokolovská 83, 18600 Praha 8, Czechoslovakia 